\title{
Early identification of DPAM in at-risk low-grade appendiceal mucinous neoplasm patients: a new approach to surveillance for peritoneal metastasis
}

\author{
Jason M. Foster ${ }^{1 *}$, Richard L. Sleightholm¹, Steve Wahlmeier ${ }^{1}$, Brian Loggie², Poonam Sharma ${ }^{3}$ and Asish Patel ${ }^{1}$
}

\begin{abstract}
Background: Disseminated peritoneal adenomucinosis (DPAM) patients often have a history of appendectomy with identification of an incidental mucinous neoplasm (low-grade appendiceal mucinous neoplasm (LAMN)). The rate of developing DPAM is not well established.

Methods: Twenty-two patients with incidental LAMN were identified and monitored with cancer markers and CT every 4-6 months. Laparoscopy with peritoneal washing was performed in patients either in the event of radiographic disease or after 12 months in absence of radiographic disease. The rate of detecting peritoneal metastasis was determined for CT scan and laparoscopy.

Results: Peritoneal metastasis was detected in $5(23 \%)$ patients. Occult disease was detected in four patients at laparoscopy without a detectable disease on CT scan. One patient developed radiographic progression at 6 months confirmed with laparoscopy. Four patients were treated with cytoreductive surgery (CRS)/HIPEC and one with CRS only. The 17 patients with negative laparoscopy remain disease free with a median follow-up of 50 months.

Conclusions: The rate of peritoneal metastasis in incidental LAMN patients was $23 \%$. Laparoscopy was the primary screening tool identifying occult metastasis. The median PCl of 7 was low, and all the patients underwent R0/R1 resections. This study revealed 1 in every 4.4 patients with LAMN may develop PMP. Longer follow-up and further patient surveillance is warranted.
\end{abstract}

Keywords: Appendiceal, Pseudomyxoma peritonei, Perforated appendicitis

\section{Background}

Appendix tumors are rare, representing less than $1 \%$ of gastrointestinal cancers and incidentally detected in approximately $1 \%$ of appendectomy specimens [1-4]. The two most common histologies identified are mucinous and carcinoid tumors [5, 6]. Carcinoid tumors, like colorectal cancer, have a propensity for lymphatic and hematogenous spread [7]. Conversely, mucinous tumors

\footnotetext{
* Correspondence: ffosterm@unmc.edu

This data was presented at the 10th International Symposium on Regional Cancer Therapies in February 2015, and we would like to submit the manuscript for publication in World Journal of Surgical Oncology.

${ }^{1}$ Division of Surgical Oncology, Department of Surgery, University of Nebraska Medical Center, 984030 Nebraska Medical Center, Omaha, NE 68198-4030, USA

Full list of author information is available at the end of the article
}

infrequently exhibit hematogenous spread, and nodal involvement is rare in low-grade mucinous tumors $[8,9]$. Peritoneal dissemination is the most common form of metastasis observed in low-grade appendiceal mucinous neoplasms (LAMN), and this condition is called pseudomyxoma peritonei (PMP). PMP has been split into lowand high-grade diseases which has been further subclassified by Ronnett into disseminated peritoneal adenomucinosis (DPAM) (low grade), peritoneal mucinous carcinomatosis (PMCA)-I (intermediate grade), and PMCA (high grade) which characterize PMP along a continuum of biological behavior [9].

Majority of patients who present with low-grade PMP are symptomatic with bulky peritoneal disease [10]. The slow-growing non-invasive biology of these 
tumors contributes to the development of extensive peritoneal disease before patients seek medical attention for clinical symptoms. This indolent pattern of growth not only affords patients with PMP an opportunity for symptom palliation but majority can achieve long-term remission with cytoreductive surgery and hyperthermic intraperitoneal chemotherapy (CRS/HIPEC) [11].

Cytoreductive procedures often require extensive operative time and extended recovery. The factors predicting long-term survival with DPAM are well established and include disease extent measured by peritoneal cancer index (PCI), ability to achieve optimal cytoreduction quantified by completeness of cytoreduction (CC) score or $\mathrm{R}$ score $(\mathrm{CC}-0 / \mathrm{CC}-1$ or $\mathrm{R} 0 / \mathrm{R} 2 \mathrm{a}$ ), and early referral to centers with experience in CRS surgery [12-14].

Optimal tumor cytoreduction can be achieved in 70$90 \%$ of DPAM patients, but this typically requires extensive cytoreductive procedures with multivisceral organ resections, prolonged hospital stay, and post-operative morbidity and mortality rates of $30-50 \%$ and $3-7 \%$, respectively [13]. Many patients who develop symptomatic low-grade PMP have a history of appendectomy with an incidental LAMN identified. Given the low incidence of LAMN tumors, there is a paucity of data that defines the rate of LAMN progression to PMP of the DPAM subtype, and like most rare tumors, there is currently no consensus on follow-up guidelines. In spite of these obstacles, the development of a surveillance strategy in LAMN may not only provide insight into the rate of PMP development but facilitate early detection of occult peritoneal metastasis in LAMN patients and improve outcomes in PMP patients. Currently, only one retrospective population study in the Netherlands reported an observed PMP incidence of $20 \%$ in 547 patients with LAMN lesions. Majority of the PMP events were detected within 24 months, but in a few cases, PMP occurred after 2 years [15]. As a result, this group recommends performing cross-sectional imaging for at least 5 years in patients with incidental LAMN tumors. Since CT imaging has known limitations in the detection of peritoneal metastasis, a second modality, diagnostic laparoscopy, was added to the surveillance regimen to determine if occult disease could be identified in patients in the absence of CT-detectable disease. Here, we report the results of this surveillance strategy.

\section{Methods}

\section{Patient population}

A retrospective chart review was conducted on patients referred for management of LAMN. In this study, lowgrade mucinous tumors were defined by the grading system reported by Carr et al. and Misrdraji et al., recently adopted by the Peritoneal Surface Oncology Group
International (PSOGI) [16-19]. All cases included in this analysis had mucin with low-grade architectural features, "pushing alteration" of the muscularis mucosa or wall, and when pathology was reviewed at our institutions, the presence of perforation, extra-appendiceal mucin, and extra-appendiceal cells was reported. Twenty-two LAMN patients were identified, diagnosed between June 2006 and May 2013, and comprehensive pathology reports were available in 20 out of the 22 patients. In two LAMN cases, the comprehensive pathology reports were not available.

\section{Surveillance strategy}

All patients had a baseline CT scan performed prior to their appendectomy, and these images were reviewed at first consultation (Fig. 1). All outside medical records and pathology and imaging reports were reviewed. Baseline tumor markers were obtained at the first consultation which included CA-19-9, CEA, and CA-125. The patients were re-evaluated every 4-6 months with clinical exams, abdomen and pelvis CT scans, and tumor markers. If any radiographic abnormality was reported by the reading radiologist consistent with concerns of peritoneal metastasis, patients were taken for diagnostic laparoscopy to pathologically document disease progression. After 12 months of surveillance, all the patients underwent diagnostic laparoscopy to determine if occult peritoneal metastasis could be identified in the absence of CT-detectable disease. At the time of laparoscopy, PCI zones were evaluated for peritoneal implantation and peritoneal washings were performed. Any suspicious nodules identified were biopsied and sent for pathological evaluation. If pathologically confirmed peritoneal disease was detected, the patient was offered definitive CRS/HIPEC. All the patients with positive findings elected to undergo CRS/HIPEC except for one patient who only underwent CRS without HIPEC.

\section{Clinical and outcomes data analysis}

Demographic information was collected on all LAMN patients including age, sex, and additional procedures performed prior to referral and any administration of chemotherapy by referring providers. The appendectomy proximal margin was negative in all 22 specimens, and in 20/22 cases, data was available to determine the presence of perforation or extra-appendiceal mucin and/or extraappendiceal cells. Following diagnostic laparoscopy, adverse events, cytology results, and rate of detection of peritoneal implants were determined. Finally, we calculated median follow-up time, overall survival, and current status of disease.

In LAMN patients where peritoneal metastasis was identified, all underwent CRS/HIPEC except for one patient who underwent CRS alone. PCI score (tumor 


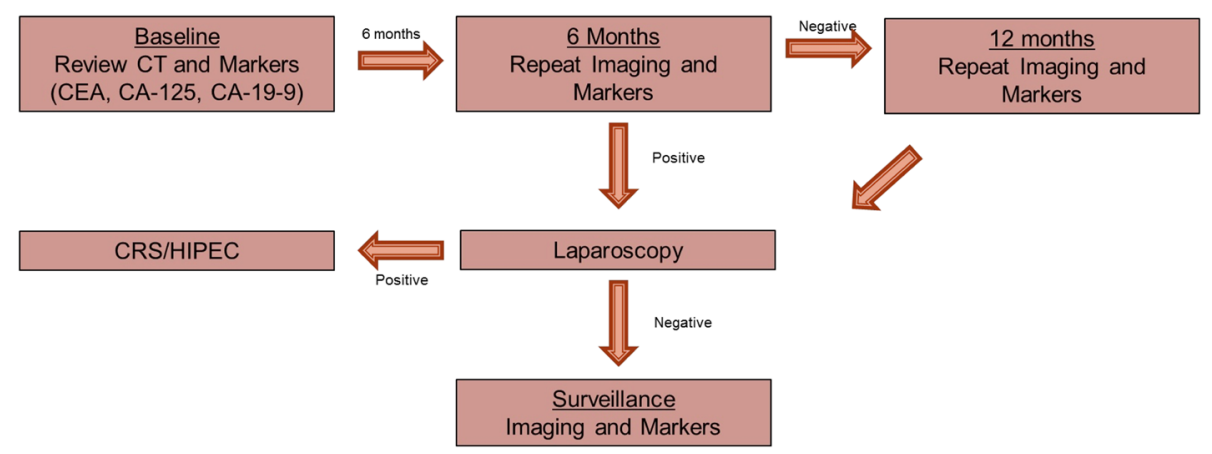

Fig. 1 Surveillance algorithm for LAMN patients

burden) [20], CC score, length of stay (LOS), overall survival, and disease-free survival status were determined. Right hemicolectomy, partial peritonectomy sites and rates, visceral organ resection rates, and total resections performed which included peritonectomy procedures were also calculated. The organ resections performed included omentectomy, right colectomy, cholecystectomy, distal pancreatectomy, splenectomy, and non-anatomical hepatectomy or liver capsule resection. All peritonectomies were limited partial resections, and the sites included the diaphragm, colic gutters, and pelvis. Morbidity and mortality were quantified by the ClavienDindo classification grades I-V [21].

\section{Results}

Demographics of surveillance group and serology results The mean age at diagnosis for the 22 LAMN patients was 53 , and $55 \%$ (12) were males. Twenty-one patients (95\%) underwent appendectomy for their disease. One patient (5\%) underwent right hemicolectomy and cholecystectomy and was also started on chemotherapy prior to referral. Following diagnostic laparoscopy, all the patients were discharged within $23 \mathrm{~h}$ of the procedure without any adverse events. In all the 22 patients, CEA, CA-19-9, and CA-125 levels were within normal limits and no patient experienced any marker elevation.

\section{LAMN appendix pathology features, rates and sites of PM implants, and cytology results}

Comprehensive pathological characteristics were available in $91 \%(20 / 22)$ of the patients (Table 1). Appendix perforation and extra-appendiceal mucin were identified in $90 \%(18 / 20)$ of the specimens. In these 18 patients with perforation and mucin extravasation, 5/18 (28\%) developed peritoneal metastasis while 13/18 (72 \%) had no evidence of peritoneal metastasis at diagnostic laparoscopy. Extra-appendiceal cells were identified in 10/20 of the patients, 4/10 (40\%) developed peritoneal metastasis while $6 / 10(60 \%)$ had no evidence of peritoneal metastasis. The overall rate of peritoneal metastasis was
5/22 (23 \%) (Fig. 2); perforation and extra-appendiceal mucin were present in $5 / 5(100 \%)$ of the patients, and extra-appendiceal cells were present in 4/5 (82\%) of the patients (Table 1). At laparoscopy, cytology was positive for cells in $60 \%(3 / 5)$ of the patients with peritoneal metastasis (Table 1 ).

The most frequent PCI zones with occult metastases were zones 0 and 1 with disease identified in all 5 patients (100\%). Figure 3 provides a comprehensive description of the sites involved for each patient and the rate of involvements in each PCI zone.

\section{Outcomes in LAMN patients without PMP}

All 17 patients had no evidence of disease or pathology on CT imaging, tumor marker serology was within normal limits, and there was no detectable tumor at laparoscopy. Tumor cytology in this group of patients was negative for tumor or mucin in peritoneal washings. One patient underwent a second laparoscopy at the 2year mark because of inflammatory changes found at the first laparoscopy, and biopsies at the first laparoscopy revealed inflammatory nodules. The second laparoscopy revealed resolution of the inflammatory changes, and no disease or inflammation was identified. The median follow-up time was 50 (20-106) months, and none of the patients developed clinical progression during the follow-up period.

\section{Outcomes in LAMN patients with PMP}

Five patients $(23 \%)$ were identified who developed peritoneal metastasis (Fig. 2). The patient that underwent right hemicolectomy (RHC) and cholecystectomy and received FOLFOX chemotherapy prior to referral had radiographic progression and evidence of DPAM at 6 months following LAMN diagnosis with evidence of omental nodularity. Laparoscopy was performed based on the CT scan and confirmed peritoneal metastasis. The radiographic PCI in this case was 8 , and the PCI at exploration was 14 (Table 2, patient \#4). In the other four cases with negative $\mathrm{CT}$ results, occult peritoneal 
Table 1 Individual descriptive appendectomy pathology and cytology results at the time of laparoscopic surveillance

\begin{tabular}{|c|c|c|c|c|}
\hline LAMN without DPAM & Perforation & Extra-appendiceal mucin & Extra-appendiceal cells & Cytology at laparoscopy \\
\hline 1 & + & + & - & - \\
\hline 2 & + & + & + & - \\
\hline 3 & + & + & + & - \\
\hline 4 & + & + & - & - \\
\hline 5 & + & + & + & - \\
\hline 6 & + & + & + & - \\
\hline 7 & + & + & + & - \\
\hline 8 & + & + & - & - \\
\hline 9 & + & + & - & - \\
\hline 10 & - & - & - & - \\
\hline 11 & + & + & - & - \\
\hline 12 & - & - & - & - \\
\hline 13 & + & + & + & - \\
\hline 14 & + & + & - & - \\
\hline 15 & + & + & - & - \\
\hline 16 & N/A & N/A & N/A & - \\
\hline 17 & N/A & $\mathrm{N} / \mathrm{A}$ & N/A & - \\
\hline Total & 13/15 (87 \%) & 13/15 (87 \%) & $6 / 15(40 \%)$ & 0/17 (0 \%) \\
\hline LAMN with DPAM & Perforation & Extra-appendiceal mucin & Extra-appendiceal cells & Cytology at laparoscopy \\
\hline 1 & + & + & + & + \\
\hline 2 & + & + & - & - \\
\hline 3 & + & + & + & - \\
\hline 4 & + & + & + & + \\
\hline 5 & + & + & + & + \\
\hline Total & $5 / 5(100 \%)$ & $5 / 5(100 \%)$ & $4 / 5(80 \%)$ & $3 / 5(60 \%)$ \\
\hline All patients & 18/20 (90 \%) & 18/20 (90 \%) & 10/20 (50 \%) & $3 / 22(14 \%)$ \\
\hline
\end{tabular}

Descriptive pathology results were not available in two patients labeled N/A

disease was identified at laparoscopy. The sites of detected disease can be found in Fig. 3.

The rate of RHC was $40 \%$ (2/5), with one performed prior to referral and one performed at the time of CRS/ HIPEC because of concerns for gross surface disease (Table 2). In both cases, the pathological analysis of the specimens revealed no surface or nodal involvement. In all cases (5/5), a CC-0/CC- 1 was achieved, and Table 2

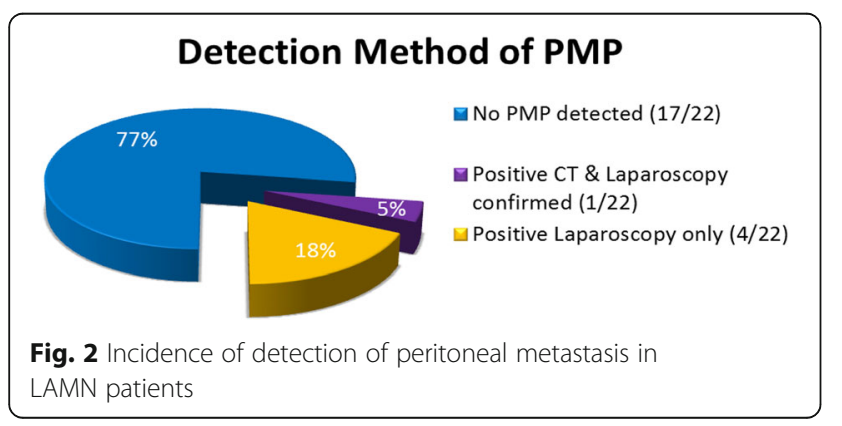

provides each patient's PCI scores, organ resections, peritonectomy rates, and LOS, while Fig. 3 provides the PCI zones of disease detection for each patient. Median PCI and total organ resection values were 7 and 3, respectively, reflecting the limited disease burden. The median LOS was 7 days, and no post-operative deaths occurred with only one patient experiencing a grade II Clavien-Dindo adverse medical event, hypertension requiring an esmolol drip.

In 4/5 patients treated with CRS/HIPEC, all the patients are alive without disease recurrence with median follow-up of 50 months. In the patient who elected to have CRS alone, disease recurrence occurred at 24 months after cytoreduction (36 months from diagnosis). The recurrence was detected on CT imaging, and the patient had no clinical symptoms. The patient underwent a second procedure with a PCI score of 19, and $\mathrm{CC}-1$ cytoreduction was achieved. The patient is currently alive without recurrence or progression. 


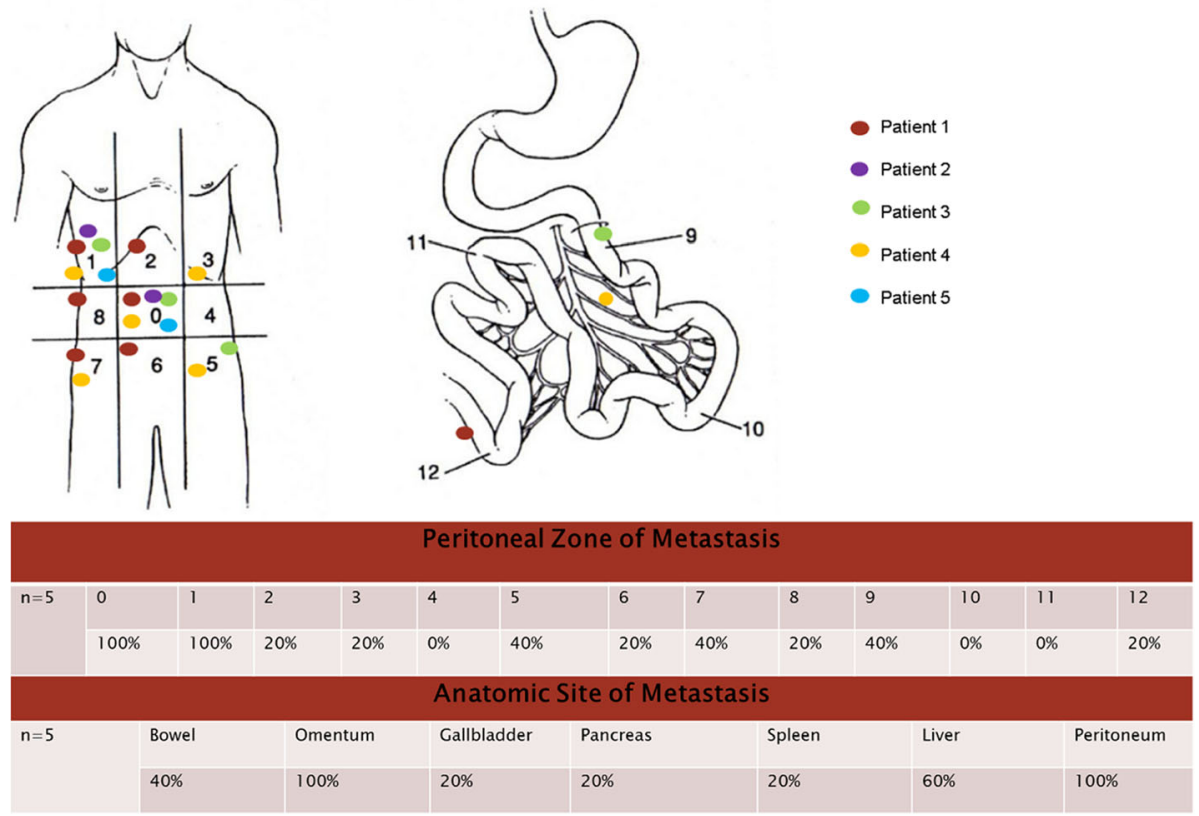

Fig. 3 Map and table of metastasis based on PCl zones

\section{Discussion}

The optimal management of incidental LAMN tumors had been a challenging area to define because of the low incidence and indolent behavior of LAMN tumors that develop peritoneal metastasis resulting in low-grade PMP, DPAM. The goal of this study was to longitudinally follow patients with only LAMN tumors, excluding all other histologies, and evaluate these patients for the development of peritoneal metastasis with imaging and laparoscopy. It has been established that low-grade mucinous appendix tumors are the most common primary tumor site resulting in the development of low-grade PMP, with very minimal risk of nodal or hematogenous dissemination [22]. What has not been clearly established is the rate at which LAMN tumors disseminate and develop low-grade PMP. In this series of 22 patients, occult peritoneal metastasis was detected in $5(23 \%)$ patients. Only one case was detected on imaging and confirmed with laparoscopy, while $80 \%(4 / 5)$ of the cases were only detected by laparoscopy. Although this is a small series, this observed rate of PMP is consistent with the rate reported by Smeenk et al., where a PMP rate of $20 \%$ was observed in patients with LAMN histology [15]. This correlation of event rates of PMP development provides support that laparoscopy may be a useful screening tool for LAMN patients given the limitations of the small sample size.

Current surveillance recommendations for the management of low-grade mucinous appendix tumors range from no routine imaging to imaging every 6-12 months, or even consideration of CRS/HIPEC for patients with perforated disease and extravasated mucin. This data revealed that a CT scan as a surveillance tool may be inadequate for the detection of early PMP. This is consistent with other data demonstrating the CT scan often understages the volume of peritoneal disease in patients with known peritoneal metastasis [19]. MRI was not performed in these patients, but recent data demonstrates it may be a more sensitive tool for detection of peritoneal metastasis [23, 24]. As a result, we have switched to

Table 2 RHC status, PCI score, organs resected, LOS, and CC score for the LAMN group with PMP

\begin{tabular}{|c|c|c|c|c|c|c|c|c|}
\hline LAMN with DPAM & $\mathrm{RHC}$ & $\mathrm{PCl}$ & \# of organs resected & Peritonectomies & LOS & CC score & HIPEC & Recurrence \\
\hline 1 & + & 8 & 2 & 2 & 7 & 1 & + & - \\
\hline 2 & - & 7 & 2 & 2 & 7 & 0 & + & - \\
\hline 3 & - & 5 & 1 & 3 & 6 & 0 & - & + \\
\hline 4 & $t^{a}$ & 14 & 2 & 3 & 8 & 1 & + & - \\
\hline 5 & - & 7 & 1 & 4 & 8 & 1 & + & - \\
\hline
\end{tabular}

All RHC specimens were negative. All peritonectomies were partial resection not complete in $\mathrm{PCl}$ zones

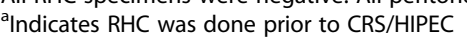


patients and only perform CT imaging when patients cannot tolerate MRI or when motion artifacts may limit interpretation.

It has been suggested in the setting of perforated appendix with extra-appendiceal mucin and/or cells in LAMN patients consider CRS/HIPEC [25]. The comprehensive pathological analysis revealed that these features were present in all patients who develop PMP; however, this feature was present in $90 \%(18 / 20)$ of all the specimens. Specifically, $13 / 18$ patients with these features were identified without detectable occult peritoneal metastasis at laparoscopy. If CRS/HIPEC was performed for all patients with perforation and extra-appendiceal mucin, $72 \%(13 / 18)$ of the patients with this feature potentially may have been over-treated and subjected to the risks of CRS/HIPEC. A similar result was observed in patients with extra-appendiceal cells where it was identified in 10/20 cases and $60 \%(6 / 10)$ of the patients would have been treated with CRS/HIPEC that did not develop DPAM. Delayed recurrences are a real concern, and continued follow-up is important. However, none of the 17 patients without evidence of occult peritoneal metastasis have developed PMP with a median follow-up period of 50 months, and they continue to undergo active imaging surveillance every 12 months.

Laparoscopy appears to be a promising screening tool in LAMN patients, but it is an invasive procedure with surgical risks. In this series, no major adverse events occurred, but bowel injury, port site infections, and hernia are complications that must be balanced with the benefits of early detection. Larger patient series will be necessary to address this question. There are limitations to this study including the small sample size and retrospective data collection. In spite of these limitations, the observed rate of PMP is consistent with the rate reported in the literature based on a study consisting 574 patients with LAMN, wherein $114(20 \%)$ developed PMP, and this provides some validation of the results observed [15]. The benefits of early detection include asymptomatic detection resulting in low PCI index at surgery, $100 \%$ optimal cytoreduction rate, and potentially a small surgical procedure evident by a low rate of organ resections. The impact on survival is not clear since the current follow-up is only 50 months, but given the favorable factors of low PCI and CC-0/CC-1 in all cases, this would support a more favorable outcome [14]. Interestingly, the only recurrence occurred in one patient who did not receive HIPEC after 3 years of follow-up. It is not clear what other factors played a role in this patient's recurrence, but this observation provides evidence that these occult tumors are biologically active and can progress/recur over time. This patient is currently in remission following the second CRS with HIPEC.
Based on this preliminary data, our group is establishing a prospective registry trial employing this surveillance protocol utilizing cross-sectional imaging (MRI or CT) every 4-6 months and serological marker assessment and offering all patients laparoscopy after 1 year of imaging surveillance or in the event of radiologic findings of peritoneal metastasis. Through a multiinstitutional collaborative effort, we hope that continued research in this area will more clearly define the incidence of PMP and result in early detection of the syndrome.

\section{Conclusions}

The rate of peritoneal metastasis in incidental LAMN patients was $23 \%$, and laparoscopy was the primary screening tool identifying occult metastasis. Given the favorable prognosis of early detection and treatment of PMP, DPAM, further trials utilizing a surveillance strategy of laparoscopy, serological markers, and crosssectional imaging are warranted.

\section{Abbreviations \\ CRS: Cytoreductive surgery; CC: Completeness of cytoreduction; \\ CT: Computed tomography; DPAM: Disseminated peritoneal \\ adenomucinosis; HIPEC: Hyperthermic intraperitoneal chemotherapy; \\ LOS: Length of stay; LAMN: Low-grade appendiceal mucinous neoplasm; \\ PCI: Peritoneal cancer index; PMCA: Peritoneal mucinous carcinomatosis; PMP: Pseudomyxoma peritonei; RHC: Right hemicolectomy;}

\section{Acknowledgements}

This research was supported by the Jonathan Platon and the Bobbie Hill Research Funds.

\section{Funding}

This research was supported by patients and patients' family donations: the Jonathan Platon and the Bobbie Hill Research Funds. The funding bodies had no role in the study design, data collection, or data interpretation.

\section{Availability of data and materials}

The datasets generated from the current study are not publicly available because in compliance with the IRB approval of this project, the data was collected, stored, and maintained on a secure server at the University of Nebraska Medical Center. De-identified data can be provided from the corresponding author on reasonable request.

\section{Authors' contributions}

JF conceived of the project and drafted the manuscript. JF and BL conducted all the surgical procedures. PS contributed to the pathologic components of the manuscript and pathology interpretation. JF, PS, RS, SW, and AP contributed to data acquisition, interpretation, and presentation, as well as drafting the manuscript. All authors read and approved the final manuscript.

\section{Competing interests \\ The authors declare that they have no competing interests.}

\section{Consent for publication}

Not applicable.

\section{Ethics approval and consent to participate}

The protocol for this research was submitted and approved by the University of Nebraska Medical Center Office of Regulatory Affairs Office's Institution Review Board. All research approved by the UMNC-IRB is conducted in accordance with HHRP 45 CRF 46 (www.unmc.edu/irb/), and this research was conducted under a retrospective (waived consent) research protocol \#647- 
12-EP. Informed consent for operative procedures was obtained from all patients.

\section{Author details}

'Division of Surgical Oncology, Department of Surgery, University of Nebraska Medical Center, 984030 Nebraska Medical Center, Omaha, NE 68198-4030, USA. 'Division of Surgical Oncology, Department of Surgery, Alegent Creighton University Health Center, Omaha, USA. ${ }^{3}$ Department of Pathology, Alegent Creighton University Health Center, Omaha, USA.

Received: 1 March 2016 Accepted: 23 August 2016

\section{Published online: 13 September 2016}

\section{References}

1. Abeloff MD, Armitage JO, Lichter AL, Niederhuber JE, eds, Abeloff's Clinical Oncology. 2nd ed. New York: NCL, Inc.; 2000;1912-1913.

2. Collins DC. 71,000 human appendix specimens. A final report, summarizing forty years' study. Am J Proctol. 1963;14:265-81.

3. Connor SJ, Hanna GB, Frizelle FA. Appendiceal tumors: retrospective clinicopathologic analysis of appendiceal tumors from 7,970 appendectomies. Dis Colon Rectum. 1998;41:75-80.

4. Nitecki SS, Wolff BG, Schlinkert R, Sarr MG. The natural history of surgically treated primary adenocarcinoma of the appendix. Ann Surg. 1994;219:51-7.

5. Gupta S, Parsa V, Adsay V, Heilbrun LK, Smith D, Shields AF, Weaver D, Philip PA, El-Rayes BF. Clinicopathological analysis of primary epithelial appendiceal neoplasms. Med Oncol. 2010;27:1073-8.

6. McGory ML, Maggard MA, Kang H, O'Connell JB, Ko CY. Malignancies of the appendix: beyond case series reports. Dis Colon Rectum. 2005;48:2264-71.

7. Moesta KT, Schlag P. Proposal for a new carcinoid tumour staging system based on tumour tissue infiltration and primary metastasis; a prospective multicentre carcinoid tumour evaluation study. West German Surgical Oncologists' Group. Eur J Surg Oncol. 1990;16:280-8.

8. Elias D, Gilly F, Quenet F, Bereder JM, Sideris L, Mansvelt B, Lorimier G, Glehen O. Pseudomyxoma peritonei: a French multicentric study of 301 patients treated with cytoreductive surgery and intraperitoneal chemotherapy. Eur J Surg Oncol. 2010;36:456-62.

9. Ronnett BM, Zahn CM, Kurman RJ, Kass ME, Sugarbaker PH, Shmookler BM Disseminated peritoneal adenomucinosis and peritoneal mucinous carcinomatosis. A clinicopathologic analysis of 109 cases with emphasis on distinguishing pathologic features, site of origin, prognosis, and relationship to "pseudomyxoma peritonei". Am J Surg Pathol. 1995;19:1390-408.

10. Flicek K, Ashfaq A, Johnson CD, Menias C, Bagaria S, Wasif N. Correlation of radiologic with surgical peritoneal cancer index scores in patients with pseudomyxoma peritonei and peritoneal carcinomatosis: how well can we predict resectability? J Gastrointest Surg. 2016;20:307-12.

11. Lord AC, Shihab O, Chandrakumaran K, Mohamed F, Cecil TD, Moran BJ. Recurrence and outcome after complete tumour removal and hyperthermic intraperitoneal chemotherapy in 512 patients with pseudomyxoma peritonei from perforated appendiceal mucinous tumours. Eur J Surg Oncol. 2015:41:396-9.

12. Chua TC, Liauw W, Zhao J, Morris DL. Upfront compared to delayed cytoreductive surgery and perioperative intraperitoneal chemotherapy for pseudomyxoma peritonei is associated with considerably lower perioperative morbidity and recurrence rate. Ann Surg. 2011;253:769-73.

13. Dayal S, Taflampas P, Riss S, Chandrakumaran K, Cecil TD, Mohamed F, Moran BJ. Complete cytoreduction for pseudomyxoma peritonei is optimal but maximal tumor debulking may be beneficial in patients in whom complete tumor removal cannot be achieved. Dis Colon Rectum. 2013;56:1366-72.

14. Jimenez W, Sardi A, Nieroda C, Sittig M, Milovanov V, Nunez M, Aydin N, Gushchin V. Predictive and prognostic survival factors in peritoneal carcinomatosis from appendiceal cancer after cytoreductive surgery with hyperthermic intraperitoneal chemotherapy. Ann Surg Oncol. 2014;21:4218-25.

15. Smeenk RM, van Velthuysen ML, Verwaal VJ, Zoetmulder FA. Appendiceal neoplasms and pseudomyxoma peritonei: a population based study. Eur J Surg Oncol. 2008;34:196-201.

16. Carr NJ, Cecil TD, Mohamed F, Sobin LH, Sugarbaker PH, Gonzalez-Moreno S, Taflampas P, Chapman S, Moran BJ. A consensus for classification and pathologic reporting of pseudomyxoma peritonei and associated appendiceal neoplasia: the results of the Peritoneal Surface Oncology Group International (PSOGI) modified Delphi process. Am J Surg Pathol. 2016;40: $14-26$.
17. Carr NJ AM, Deans GT, Sobin LH. Chapter 5 Tumours of Appendix: WHO Classification of tumors. Pathology and Genetics of the digestive system. In: Altonen Ha, editors. France: IARC Press, International Agency for Research on Cancer 69372 Lyon; 2000;91-100.

18. Misdraji J. Appendiceal mucinous neoplasms: controversial issues. Arch Pathol Lab Med. 2010;134:864-70.

19. Misdraji J, Yantiss RK, Graeme-Cook FM, Balis UJ, Young RH. Appendiceal mucinous neoplasms: a clinicopathologic analysis of 107 cases. Am J Surg Pathol. 2003:27:1089-103.

20. Jacquet $\mathrm{P}$, Sugarbaker $\mathrm{PH}$. Clinical research methodologies in diagnosis and staging of patients with peritoneal carcinomatosis. Cancer Treat Res. 1996; 82:359-74.

21. Dindo D, Demartines N, Clavien PA. Classification of surgical complications: a new proposal with evaluation in a cohort of 6336 patients and results of a survey. Ann Surg. 2004:240:205-13.

22. Gonzalez-Moreno S, Brun E, Sugarbaker PH. Lymph node metastasis in epithelial malignancies of the appendix with peritoneal dissemination does not reduce survival in patients treated by cytoreductive surgery and perioperative intraperitoneal chemotherapy. Ann Surg Oncol. 2005;12:72-80.

23. Low RN, Barone RM, Lacey C, Sigeti JS, Alzate GD, Sebrechts CP. Peritoneal tumor: MR imaging with dilute oral barium and intravenous gadoliniumcontaining contrast agents compared with unenhanced MR imaging and CT. Radiology. 1997;204:513-20.

24. Yan TD, Sim J, Morris DL. Selection of patients with colorectal peritoneal carcinomatosis for cytoreductive surgery and perioperative intraperitoneal chemotherapy. Ann Surg Oncol. 2007:14:1807-17.

25. Stocchi L, Wolff BG, Larson DR, Harrington JR. Surgical treatment of appendiceal mucocele. Arch Surg. 2003:138:585-9.

\section{Submit your next manuscript to BioMed Central and we will help you at every step:}

- We accept pre-submission inquiries

- Our selector tool helps you to find the most relevant journal

- We provide round the clock customer support

- Convenient online submission

- Thorough peer review

- Inclusion in PubMed and all major indexing services

- Maximum visibility for your research

Submit your manuscript at www.biomedcentral.com/submit
Biomed Central 